\title{
Bayesian Changepoint Detection Through Switching Regressions: Contact Point Determination in Material Indentation Experiments
}

\section{Citation}

Yuen, Shelten G., Daniel G. Rudoy, Robert D. Howe, and Patrick J. Wolfe. 2007. Bayesian changepoint detection through switching regressions: Contact point determination in material indentation experiments. In Proceedings, IEEE/SP 14th Workshop on Statistical Signal Processing: August 25-29, 2007, Madison, WI, ed. Institute of Electrical and Electronics Engineers, 104-108. Piscataway, NJ: IEEE.

\section{Published Version}

doi:10.1109/SSP.2007.4301227

\section{Permanent link}

http://nrs.harvard.edu/urn-3:HUL.InstRepos:22085978

\section{Terms of Use}

This article was downloaded from Harvard University's DASH repository, and is made available under the terms and conditions applicable to Other Posted Material, as set forth at http:// nrs.harvard.edu/urn-3:HUL.InstRepos:dash.current.terms-of-use\#LAA

\section{Share Your Story}

The Harvard community has made this article openly available.

Please share how this access benefits you. Submit a story.

Accessibility 


\title{
BAYESIAN CHANGEPOINT DETECTION THROUGH SWITCHING REGRESSIONS: CONTACT POINT DETERMINATION IN MATERIAL INDENTATION EXPERIMENTS
}

\author{
Shelten G. Yuen, Daniel Rudoy, Robert D. Howe, and Patrick J. Wolfe \\ School of Engineering and Applied Sciences \\ Department of Statistics, Harvard University \\ Harvard-MIT Division of Health Sciences \& Technology \\ Oxford Street, Cambridge, MA 02138 USA \\ \{sgyuen, rudoy, howe, patrick\}eseas.harvard.edu
}

\begin{abstract}
Material indentation is a popular method for determining the mechanical properties of biomaterials. The basic premise of an indentation experiment is to physically displace the sample using an indenter that measures resistive force, in order to formulate a force-displacement curve. However, doing so requires estimating the initial contact event between the indenter and the sample-a statistical changepoint detection problem that has not been rigorously addressed in the biomaterials literature to date. Here we adopt a hierarchical Bayesian approach to contact point determination based on switching regressions, which generalizes an algorithm popular with practitioners and enables both hyperparameter estimation as well as uncertainty quantification. Results using several experimentally obtained silicone indentation data sets indicate that our approach outperforms existing techniques.
\end{abstract}

Index Terms - changepoint detection, switching regressions, Bayesian estimation, material indentation, soft tissue mechanics

\section{INTRODUCTION}

In this paper we apply a hierarchical Bayesian approach for changepoint detection to the problem of estimating contact points in material indentation studies. Indentation is a popular technique for probing materials to analyze their mechanical properties and has been used to characterize a wide range of physiological systems at different scales. Applications on the microscopic scale include studying the stiffness of cells by indenting their surfaces with an atomic force microscope [1]. More recently, indentation has been used at a macroscopic level to characterize the response of liver tissue to the high strains experienced in surgical manipulation [2]. The potential exists to apply this technique to clinically diagnose tissue disease, such as cardiac hypertrophy [3].

The basic premise of an indentation experiment is to physically displace the sample using an indenter that concurrently measures resistive force. Typically, the indenter is moved from a noncontact position toward and into the sample, yielding a measurement of force for every indenter position. These data are subsequently converted to a force-displacement curve by discarding the measurements occurring prior to the initial contact point. This curve can then be used to infer the material properties of the sample, in a manner analogous to estimating the stiffness of a spring compressed a specified distance by a force of known magnitude.

An accurate determination of this initial contact point is critical for inferring material properties. Indeed, errors in contact point estimation on the order of $5 \mathrm{~nm}$ for a $2.7 \mu \mathrm{m}$ sample can cause an increase of nearly $200 \%$ in the estimated Young's modulus (a material property related to stiffness) for small displacements of thin films [4]. Moreover, measuring the contact point during indentation of soft materials is often difficult or impossible (as, e.g., the contact sensors do not exist on the size scale of the experiment, or cannot operate in fluids). Instead, researchers estimate the contact point by analyzing the force and indenter position data directly.

Visual inspection and manual thresholding methods are often used to determine contact points, but are prone to errors. An alternative approach is to obtain a mechanics-derived polynomial relationship between force and displacement [5] and use it to jointly estimate both the contact point and the Young's modulus of the sample [1]. However, this technique relies on a number of physical assumptions of dubious generality $[1,4]$. The shortcomings of these existing methods are a strong motivator for the application of a robust statistical procedure. In this vein, an approach recently introduced in [6] is to find the changepoint that minimizes the mean-squared error of a linear pre-contact and quadratic post-contact fit to the raw indentation data, and to declare this point estimate as the initial contact point. In [6], this method is termed the bidomain polynomial (BDP) model and is implemented via an exhaustive search; however, it is fitted under the assumption that the pre- and post-contact likelihood precision parameters are equal-an assumption which is unlikely to hold in prac- 
tice, owing to the vibrational dampening effects that occur through contact with the sample. More generally, all of the above methods fail to provide a means of quantifying uncertainty in changepoint estimation.

To correct these shortcomings, we adopt a hierarchical Bayesian framework for switching regressions [7] and extend it to appropriately model the changepoint problem at hand. Through this approach, contact point estimation is realized by posterior sampling via standard Monte Carlo techniques, enabling both hyperparameter estimation and uncertainty quantification. Not only does this more flexible approach recover the aforementioned BDP model as a special case, but it is also applicable to a wider variety of material indentation systems and indenter geometries. Following validation with synthetic data, we show that our framework outperforms the BDP method when using data collected in a set of real indentation experiments. The proposed model is described in Section 2, and an accompanying Gibbs sampling framework is described in Section 3. In Section 4 we present experimental results; we conclude with a discussion of ongoing and future work in Section 5.

\section{PROBLEM STATEMENT AND MODEL ELICITATION}

During an indentation experiment, the indenter moves continuously through a sequence of $N$ measured positions $\boldsymbol{x}=$ $\left(x_{1}, x_{2}, \ldots, x_{N}\right)$ and records a force measurement (in Newtons) $y_{i}$ at every position (in millimeters) $x_{i}$, resulting in a corresponding sequence of force measurements. Let $k$ denote the position index at which the indenter first makes contact with the sample, so that $x_{k}$ denotes the first measured position after which contact is made. Henceforth we refer to the index $k$ as the contact point-or equivalently, changepoint in the switching regression we formulate as follows. In keeping with the common Hertz model for a conically-tipped indenter [5], we assume that $\boldsymbol{y}$ is a linear (pre-contact) and then quadratic (post-contact) function of $\boldsymbol{x}$ with unknown regression parameters and additive Gaussian noise terms:

$$
y_{i} \sim \begin{cases}\mathcal{N}\left(\alpha_{1}+\alpha_{2} x_{i}, \sigma_{1}^{2}\right) & \text { when } \quad 1 \leq i \leq k \\ \mathcal{N}\left(\beta_{1}+\beta_{2} x_{i}+\beta_{3} x_{i}^{2}, \sigma_{2}^{2}\right) & \text { when } \quad k<i<N\end{cases}
$$

where the parameters $\left\{\alpha_{1}, \alpha_{2}\right\}$ and $\left\{\beta_{1}, \beta_{2}, \beta_{3}\right\}$ are the coefficients of the linear and quadratic models, respectively, and $\sigma_{1}^{2}$ and $\sigma_{2}^{2}$ denote the respective noise variances. We note that this assumption is motivated physically by the fact that the characteristics of the "noise" are altered when the indenter makes contact with the sample. ${ }^{1}$

To perform inference in this switching regressions scenario, we adopt and extend the hierarchical Bayesian approach of [7]. This requires the specification of prior distributions for

\footnotetext{
${ }^{1}$ For instance, noise variances are reduced owing to friction between the indenter and the sample-in our experiments by as much as $60 \%$.
}

all model parameters, including the changepoint index $k$, both sets of regression coefficients $\left\{\alpha_{1}, \alpha_{2}\right\}$ and $\left\{\beta_{1}, \beta_{2}, \beta_{3}\right\}$, and the noise variances $\sigma_{1}^{2}, \sigma_{2}^{2}$. In particular, let $\boldsymbol{\theta}_{1}=\left(\alpha_{1}, \alpha_{2}\right)^{T}$ and $\boldsymbol{\theta}_{2}=\left(\beta_{1}, \beta_{2}, \beta_{3}\right)^{T}$ represent the pre- and post-contact regression coefficients. Then we assume that $\boldsymbol{\theta}_{2} \in \mathbb{R}^{3} \sim$ $\mathcal{N}\left(\boldsymbol{\theta}_{0}, \boldsymbol{\Sigma}\right)$ and $\boldsymbol{\theta}_{1} \in \mathbb{R}^{2} \sim \mathcal{N}\left(\tilde{\boldsymbol{\theta}}_{0}, \tilde{\boldsymbol{\Sigma}}\right)$; a prior on $\boldsymbol{\theta}_{1}$ will be obtained by marginalizing over the third element of $\boldsymbol{\theta}_{2}$. A standard inverse-gamma conjugate prior $\mathcal{I G}\left(a_{0}, b_{0}\right)$ is adopted for both noise variances $\sigma_{1}^{2}$ and $\sigma_{2}^{2}$. Owing to sensitivity to hyperparameters, we follow standard practice and adopt hyperpriors for increased model robustness. In particular, a normal-Wishart prior is assumed on $\left(\boldsymbol{\theta}_{0}, \boldsymbol{\Sigma}\right)$ so that $\boldsymbol{\theta}_{0} \sim$ $\mathcal{N}(\boldsymbol{\mu}, \boldsymbol{C})$ and $\boldsymbol{\Sigma} \sim \mathcal{I} \mathcal{W}(\rho \boldsymbol{V}, \rho)$. A gamma prior is assumed on $b_{0}$ so that $b_{0} \sim \mathcal{G}\left(\epsilon_{1}, \epsilon_{2}\right)$.

Thus, we have specified a 21-parameter hierarchical model with parameters $\boldsymbol{\phi} \triangleq\left(\boldsymbol{\mu}, \boldsymbol{C}, \boldsymbol{V}, \rho, a_{0}, \epsilon_{1}, \epsilon_{2}\right)$ assumed to be deterministic and known. The posterior distribution of all the model parameters conditioned on the data and constants may then be obtained as

$$
\begin{aligned}
& p\left(k, \boldsymbol{\theta}_{1}, \boldsymbol{\theta}_{2}, \sigma_{1}^{2}, \sigma_{2}^{2}, \boldsymbol{\theta}_{0}, \boldsymbol{\Sigma}, b_{0} \mid \boldsymbol{y}, \boldsymbol{\phi}\right) \propto p\left(\boldsymbol{y} \mid k, \boldsymbol{\theta}_{1}, \boldsymbol{\theta}_{2}, \sigma_{1}^{2}, \sigma_{2}^{2}\right) \\
& \times p\left(k \mid \boldsymbol{\theta}_{1}, \boldsymbol{\theta}_{2}, \sigma_{1}^{2}, \sigma_{2}^{2}\right) p\left(\boldsymbol{\theta}_{1}, \boldsymbol{\theta}_{2} \mid \boldsymbol{\theta}_{0}, \boldsymbol{\Sigma}, \boldsymbol{\phi}\right) p\left(\boldsymbol{\theta}_{\mathbf{0}} \mid \boldsymbol{\mu}, \boldsymbol{C}\right) \\
& \times p(\boldsymbol{\Sigma} \mid \rho, \boldsymbol{V}) p\left(\sigma_{1}^{2} \mid a_{0}, b_{0}\right) p\left(\sigma_{2}^{2} \mid a_{0}, b_{0}\right) p\left(b_{0} \mid \epsilon_{1}, \epsilon_{2}\right),
\end{aligned}
$$

from which inference about the changepoint index $k$ follows directly from the marginal posterior $p(k \mid \boldsymbol{y}, \boldsymbol{\phi})$. A number of standard estimators are available given (samples from) this distribution; in particular, the maximum a posteriori (MAP) estimate corresponds to a generalized version of the BDP method [3], which selects the changepoint that minimizes the squared error of a linear pre-contact and quadratic post-contact fit to the data.

\section{INFERENCE VIA GIBBS SAMPLING}

The high dimensionality of the parameter space in this hierarchical model suggests a simulation-based approach to inference; indeed, it is by now standard to use Markov chain Monte Carlo methods to draw samples from the posterior in such cases. We generalized the Gibbs sampler proposed in [7] by using additional hyperpriors and allowing for arbitrary polynomial regressions to each side of the changepoint. In the below we specify the modified sampler as it pertains to our linear-quadratic switching regressions formulation, maintaining the notation of [7] for convenience. Owing to the fact that pre- and post-changepoint model orders are fixed (albeit unequal), a reversible-jump sampler is not required.

To specify the conditional distributions required for Gibbs sampling, we first define the design matrices $\boldsymbol{X}_{1}^{(k)}, \boldsymbol{X}_{2}^{(k)}$ for $k$ fixed as

$$
\boldsymbol{X}_{1}^{(k)}=\left[\begin{array}{cccc}
1 & 1 & \ldots & 1 \\
x_{1} & x_{2} & \ldots & x_{k}
\end{array}\right]^{T}, \boldsymbol{X}_{2}^{(k)}=\left[\begin{array}{cccc}
1 & 1 & \ldots & 1 \\
x_{k+1} & x_{k+2} & \ldots & x_{N} \\
x_{k+1}^{2} & x_{k+2}^{2} & \ldots & x_{N}^{2}
\end{array}\right]^{T}
$$


along with the corresponding pre- and post-changepoint partitions $\boldsymbol{Y}_{1}^{(k)}=\left[y_{1}, \ldots, y_{k}\right]^{T}$ and $\boldsymbol{Y}_{2}^{(k)}=\left[y_{k+1}, \ldots, y_{N}\right]^{T}$. Finally, let $\boldsymbol{\Theta}=\left\{k, \boldsymbol{\theta}_{1}, \boldsymbol{\theta}_{2}, \sigma_{1}, \sigma_{2}, \boldsymbol{\theta}_{0}, \boldsymbol{\Sigma}, b_{0}\right\}$ denote the set of all non-deterministic model parameters, and define the residual vector $\boldsymbol{Z}_{i}^{(k)}$ for $i \in\{1,2\}$ as $\boldsymbol{Z}_{i}^{(k)}=\boldsymbol{Y}_{i}^{(k)}-\boldsymbol{X}_{i}^{(k)} \boldsymbol{\theta}_{i}$. Standard manipulations then yield the following full conditional distributions of the form $p\left(z \mid \boldsymbol{\Theta}_{-z}, \boldsymbol{\phi}, \boldsymbol{y}\right)$, where $\boldsymbol{\Theta}_{-z}$ denotes $\boldsymbol{\Theta} \backslash z$ and $i \in\{1,2\}$ :

$$
\begin{aligned}
& p\left(k \mid \boldsymbol{\Theta}_{-k}, \boldsymbol{y}\right) \propto \sigma_{1}^{-k} \sigma_{2}^{-(n-k)} \exp \left(-\sum_{i=1}^{2} \frac{\boldsymbol{Z}_{i}^{(k) T} \boldsymbol{Z}_{i}^{(k)}}{2 \sigma_{i}^{2}}\right) \\
& p\left(\boldsymbol{\theta}_{i} \mid \boldsymbol{\Theta}_{-\boldsymbol{\theta}_{i}}, \boldsymbol{y}\right)=\mathcal{N}\left(\boldsymbol{B}_{i}^{(k)} \boldsymbol{b}_{i}^{(k)}, \boldsymbol{B}_{i}^{(k)}\right) \\
& p\left(\sigma_{i}^{2} \mid \boldsymbol{\Theta}_{-\sigma_{i}}, \boldsymbol{y}, \boldsymbol{\phi}\right)=\mathcal{I} \mathcal{G}\left(a_{0}+\frac{k}{2}, \frac{\boldsymbol{Z}_{i}^{(k) T} \boldsymbol{Z}_{i}^{(k)}}{2}+b_{0}\right) \\
& p\left(b_{0} \mid \boldsymbol{\theta}_{-b_{0}}, \boldsymbol{\phi}\right)=\mathcal{G}\left(\epsilon_{1},\left(\epsilon_{2}^{-1}+\sigma_{1}^{-2}+\sigma_{2}^{-2}\right)^{-1}\right) \\
& p\left(\boldsymbol{\theta}_{0} \mid \boldsymbol{\Theta}_{-\boldsymbol{\theta}_{0}}, \boldsymbol{\phi}\right)=\mathcal{N}(\boldsymbol{\delta}, \boldsymbol{\Delta}) \\
& p\left(\boldsymbol{\Sigma} \mid \boldsymbol{\Theta}_{-\boldsymbol{\Sigma}}, \boldsymbol{\phi}\right)=\mathcal{I} \mathcal{W}(\boldsymbol{\Lambda}, \rho+1)
\end{aligned}
$$

Beginning with (2), we define $\boldsymbol{B}_{i}^{(k)}=\left(\sigma_{i}^{-2} \boldsymbol{X}_{i}^{(k) T} \boldsymbol{X}_{i}^{(k)}+\right.$ $\left.\tilde{\boldsymbol{\Sigma}}_{i}^{-1}\right)^{-1}$ and $\boldsymbol{b}_{i}^{(k)}=\sigma_{i}^{-2} \boldsymbol{X}_{i}^{(k) T} \boldsymbol{Y}_{i}^{(k)}+\tilde{\boldsymbol{\Sigma}}_{i}^{-1} \tilde{\boldsymbol{\theta}}_{0}$ for $i \in\{1,2\}$, with $\tilde{\boldsymbol{\Sigma}}_{1}^{-1}=\left(\boldsymbol{\Sigma}^{-1}\right)_{1: 2,1: 2}, \tilde{\boldsymbol{\Sigma}}_{2}^{-1}=\boldsymbol{\Sigma}^{-1}$, and $\tilde{\boldsymbol{\theta}}_{0}$ denoting the first two elements of $\boldsymbol{\theta}_{0}$. The covariance and mean terms of (4) are given respectively by $\boldsymbol{\Delta}=\left(\boldsymbol{C}^{-1}+2 \boldsymbol{\Sigma}^{-1}\right)^{-1}$ and $\boldsymbol{\delta}=\boldsymbol{\Delta}\left(\boldsymbol{C}^{-1} \boldsymbol{\mu}+\boldsymbol{\Sigma}^{-1}\left(\tilde{\boldsymbol{\theta}}_{1}+\boldsymbol{\theta}_{2}\right)\right)$, with $\tilde{\boldsymbol{\theta}}_{1}=\boldsymbol{\theta}_{1} \cup\{0\}$. Finally, the inverse-Wishart parameter matrix $\Lambda$ in (5) is defined by $\boldsymbol{\Lambda}=\rho \boldsymbol{V}+\left(\tilde{\boldsymbol{\theta}}_{1}-\boldsymbol{\theta}_{0}\right)\left(\tilde{\boldsymbol{\theta}}_{1}-\boldsymbol{\theta}_{0}\right)^{T}+\left(\boldsymbol{\theta}_{2}-\boldsymbol{\theta}_{0}\right)\left(\boldsymbol{\theta}_{2}-\boldsymbol{\theta}_{0}\right)^{T}$.

Sampling these model parameters one at a time, each conditional upon the others, defines a Gibbs sampler with respect to $p(\boldsymbol{\Theta} \mid \boldsymbol{y}, \boldsymbol{\phi})$. Consider such a sampler initialized to $\boldsymbol{\Theta}^{(0)}=\left(k^{(0)}, \boldsymbol{\theta}_{1}^{(0)}, \boldsymbol{\theta}_{2}^{(0)}, \sigma_{1}^{2^{(0)}}, \sigma_{2}^{2(0)}, \boldsymbol{\theta}_{0}^{(0)}, \boldsymbol{\Sigma}^{(0)}, b_{0}^{(0)}\right)$ and in state $\Theta^{(m-1)}$ after $m-1$ iterations; the next iteration then proceeds as follows:

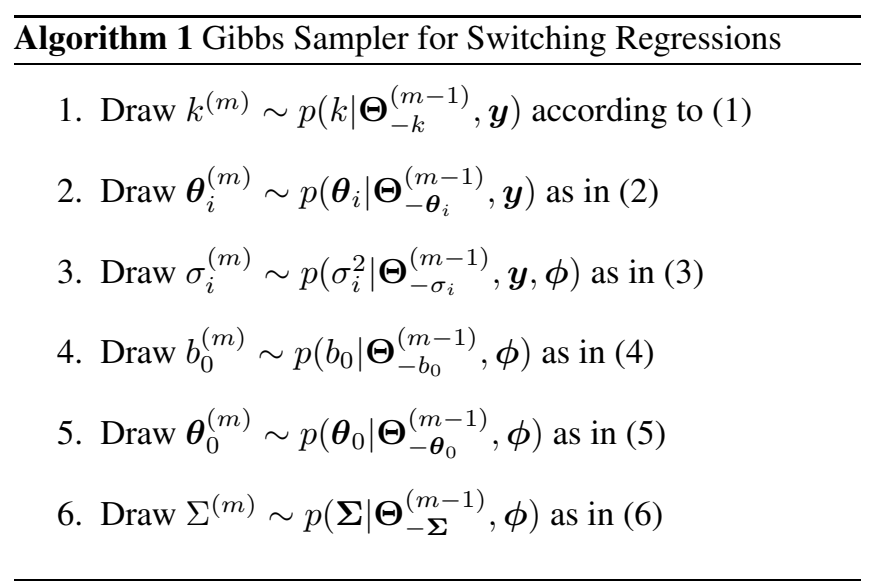

After $M \gg 1$ iterations, the resultant collection of samples is approximately distributed according to the joint posterior distribution $p\left(k, \boldsymbol{\theta}_{1}, \boldsymbol{\theta}_{2}, \sigma_{1}^{2}, \sigma_{2}^{2}, \boldsymbol{\theta}_{0}, \boldsymbol{\Sigma}, b_{0} \mid \boldsymbol{y}, \boldsymbol{\phi}\right)$.

\section{VALIDATION AND EXPERIMENTAL RESULTS}

After successful validation using synthetic data generated according to the model of Section 3, we proceeded to test Algorithm 1 on experimental data. Here, precise measurements of contact points were available owing to an impedance-measuring electrode mounted on the indenter. ${ }^{2}$ Ten indentation experiments were conducted in which data were collected by indenting a soft silicone sample (Aquaflex, Parker Laboratories, Fairfield, NJ) using the methods of [2]. This experiment is of interest because the sample is similar to those used as proxies for human tissue in material indentation studies [8]. A metal hemi-spherical indenter compressed the sample at $10 \mathrm{~mm} / \mathrm{s}$, and the resulting forces were measured to yield data as shown in Figure 1. The sample was roughly $20 \mathrm{~mm}$ in depth, and the maximum indentation was approximately $7 \mathrm{~mm}$; each experiment provided $\approx 960$ data points.

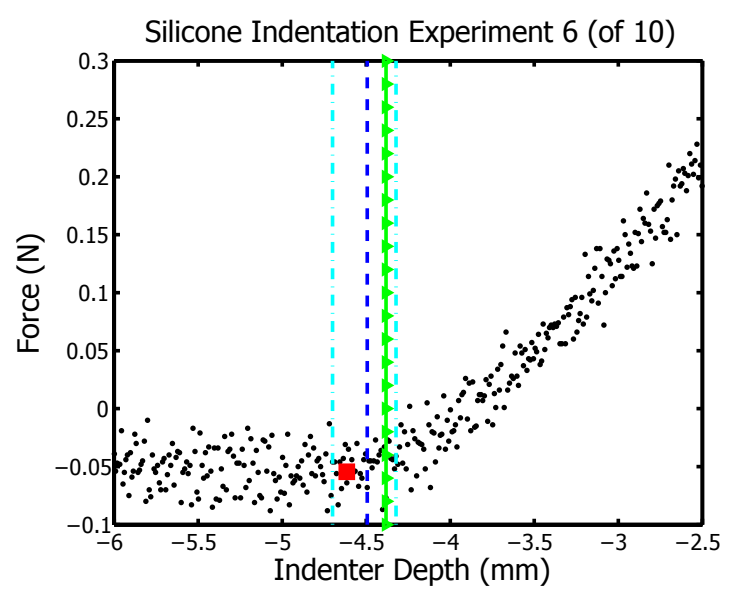

Fig. 1. Experiment 6: Indentation data (black) with truth (red 口), MMSE estimate (blue - -), 95\% posterior interval (cyan $-.-)$, and BDP estimate (green $\triangleright$ ).

The Gibbs sampler of Section 3 was then employed to evaluate the efficacy of the proposed switching regressions model, with the vector $\phi$ of fixed parameters set as follows: $\boldsymbol{\mu}=(0,0,0)^{T}, \boldsymbol{C}^{-1}=\mathbf{0}, \boldsymbol{V}^{-1}=\mathbf{0}, \rho=2, a_{0}=2, \epsilon_{1}=$ $1.0, \epsilon_{2}=.001$. Approximate knowledge of the initial distance between the indenter and sample permitted the use of a discrete uniform prior $U(125,250)$ for $k$. It is well known that the physics of the post-contact response can differ between small and large deformations, thereby requiring the use of multiple post-contact models to analyze the entire experiment. Instead we truncate the data to $N=450$ and focus on modeling the small-deformation regime; joint analysis of short and large deformation data is the subject of ongoing work. For each of the ten truncated data sets, the Gibbs sampler was executed for 100,000 iterations; convergence was monitored using standard techniques. All but the first 1000

\footnotetext{
${ }^{2}$ Our purpose here is to experimentally verify the model under studyobtaining the true contact point by direct measurement in this manner is not possible for all scales and testing environments of interest.
} 


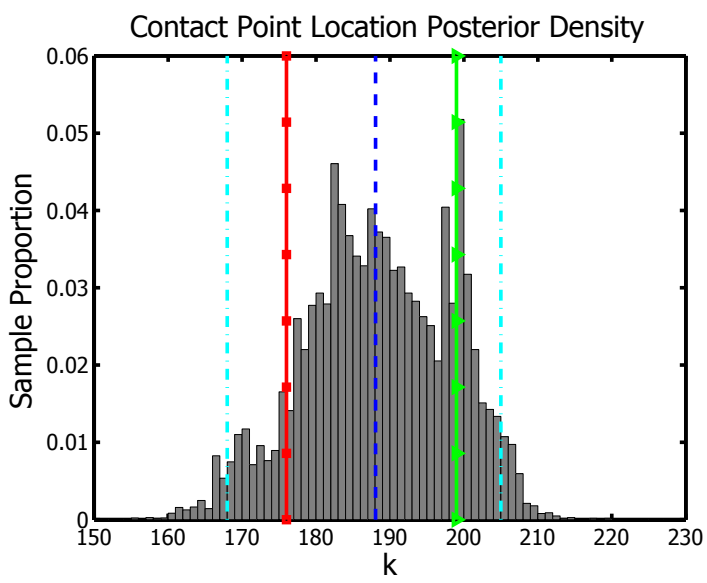

Fig. 2. Experiment 6: Posterior density of the contact point $k$ (gray) with truth (red $\mathbf{\square}$ ), MMSE estimate (blue --), 95\% posterior interval (cyan -.- ), and BDP estimate (green $\bullet$ )

samples of each run were used to estimate the marginal posterior densities, from which minimum mean-square error (MMSE) point estimates and $95 \%$ posterior intervals were obtained. BDP was also implemented and run with a restriction of $k \in$ $\{125,126, \ldots, 250\}$ to enable an equal comparison.

Prior to describing the aggregate results of our ten silicone indentation experiments, we first discuss a representative example in detail. As illustrated in Figure 1 for Experiment 6 , the posterior mean estimate of the contact point, $k=188$, is in closer agreement with the measured ground truth of $k=176$ than the BDP (approximate MAP) estimate of $k=199$. The estimated marginal posterior density of the changepoint index is shown in Figure 2; its diffuse nature underscores the importance of uncertainty quantification in a Bayesian framework, and its multimodality casts doubt on appropriateness of an approach based on MAP estimation.

A similar line of reasoning is suggested by examining the BDP objective function shown in Figure 3. The curve is nearly flat in the regions where the marginal posterior density of the changepoint has most of its mass. Moreover, the noisy data gives rise to spurious minima in the objective functionwhich in turn provides a strong motivation for explicitly modeling the noise, a key feature of the proposed hierarchical Bayesian framework. Estimates of the pre- and post-contact noise variances for Experiment 6 are shown in Figure 4; the latter appears smaller because the dampening effect of friction reduces indenter jitter.

A summary of results from the hierarchical Bayesian and BDP methods is given in Table 1. We observe that on average the Bayesian approach has significantly better point estimate performance; across all ten experiments the average error incurred was 7.4 positions (or $0.07 \mathrm{~mm}$ depth), in contrast to an average error of 9.2 positions (or $0.09 \mathrm{~mm}$ depth) for the BDP method-an improvement of $20 \%$. Furthermore, the Bayesian method appears less prone to large estimation

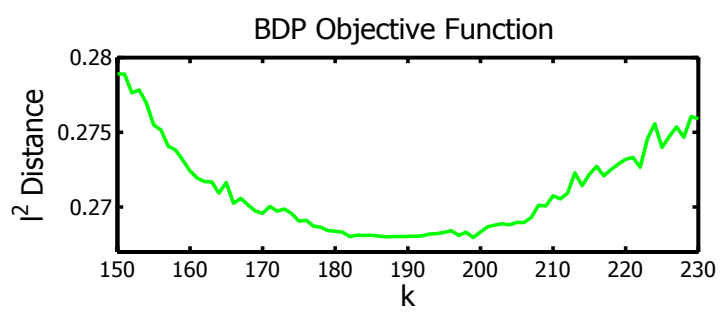

Fig. 3. Experiment 6: BDP objective function ( $\ell^{2}$ distance) evaluated over $k$

\begin{tabular}{|c|c|c|c|c|c|c|}
\hline No. & Truth & $2.5 \%$ & Med & $97.5 \%$ & $\begin{array}{l}\text { MMSE } \\
\text { Error }\end{array}$ & $\begin{array}{l}\text { BDP } \\
\text { Error }\end{array}$ \\
\hline 1 & 170 & 161 & 178 & 193 & -8.5 & -2.0 \\
\hline 2 & 186 & 170 & 190 & 205 & -3.9 & -13.0 \\
\hline 3 & 184 & 178 & 198 & 213 & -13.6 & -8.0 \\
\hline 4 & 181 & 169 & 184 & 207 & -5.2 & 2.0 \\
\hline 5 & 179 & 154 & 200 & 209 & -17.3 & -25.0 \\
\hline 6 & 176 & 168 & 188 & 205 & -12.0 & -23.0 \\
\hline 7 & 176 & 166 & 181 & 195 & -4.7 & 0.0 \\
\hline 8 & 185 & 166 & 190 & 201 & -3.6 & -10.0 \\
\hline 9 & 179 & 157 & 178 & 195 & 1.6 & -3.0 \\
\hline 10 & 176 & 167 & 178 & 200 & -3.2 & -6.0 \\
\hline \multicolumn{5}{|c|}{ Average Error } & -7.0 & -8.8 \\
\hline \multicolumn{5}{|c|}{ Standard Deviation } & 5.7 & 9.2 \\
\hline
\end{tabular}

Table 1. Summary of contact point estimation on 10 silicone indentation experiments.

errors such as those incurred by BDP in Experiments 4 and 5; this contributes to the reduced estimation error variance for the Bayesian method. Finally, note that the $95 \%$ posterior interval always includes the true contact point.

Finally, it is important to discuss the necessity of using an informative prior on the location of the changepoint, as we have done here. Consider the marginal posterior of the changepoint for Experiment 5 with the prior on $k$ set to $k \sim$ $U(1,450)$, as shown in Figure 5. The resultant bimodal distribution suggests that two disparate sets of contact point solutions exist to explain the linear-to-quadratic model: the leftmost mode corresponds to a set of fits in which the quadratic function rises slowly (implying a less stiff sample); the rightmost mode corresponds to fits where the quadratic function rises quickly (implying a more stiff sample). However, the approximate distance from the initial position of the indenter to the sample is known in the case at hand, and precludes the possibility of the changepoint occurring in the vicinity of the left mode, thus motivating the use of a prior on $k$ that restricts the support to admissible changepoint locations.

We surmise that model misspecification is the main reason for failure of the BDP method; deviations from the assumed noise statistics, as well as the assumption of equal noise variances both pre- and post-contact, are apt to cause large estimation errors. In contrast, the Bayesian switching regressions 


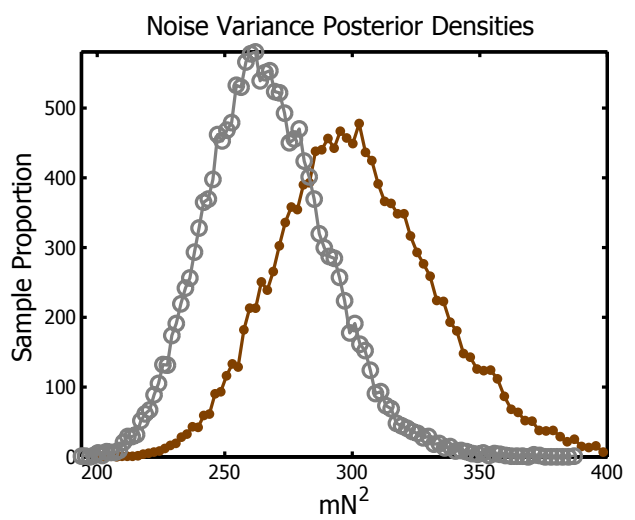

Fig. 4. Experiment 6: Posterior densities of pre- and postcontact model noise variances $\sigma_{1}^{2}$ (gray o-),$\sigma_{2}^{2}$ (brown .-)

model we propose not only accounts for a pre-to-post-contactpoint change in noise variances, but also provides a full posterior distribution for uncertainty quantification as well as point estimates of these and other model parameters.

\section{DISCUSSION}

In this work, we developed and applied a hierarchical Bayesian method to the problem of contact point estimation in material indentation experiments. Studies using experimental silicone data showed the proposed method to have significantly improved point estimates over the prevailing bi-domain polynomial fitting technique of [6], along with accompanying measures of uncertainty. Additionally, this Bayesian framework directly provides estimates of physically-relevant parameters in the system, including the pre- and post-contact noise variances as well as the regression coefficients from which stiffness of the material may subsequently be inferred. Our study demonstrates that the marginal posterior distribution of the contact point is diffuse and multimodal—strongly underscoring the need for uncertainty quantification.

Contact point determination is crucial to the accurate characterization of material properties in indentation experiments. For many materials, polynomial post-contact models are appropriate; however, a number of biomaterials have complicated post-contact responses that are better described with non-parametric models. Future work will investigate the use of non-parametric regression, as well as a model extension to multiple changepoints in post-contact to allow for the concurrent consideration of data collected through small and large deformation regimes.

Acknowledgments: The authors would like to thank Amy Kerdok for collection of the experimental data. The first author is sponsored by United States National Institutes of Health grant no. NIH R01 HL073647-01. The second author is sponsored by the National Defense Science and Engineering Graduate Fellowship.

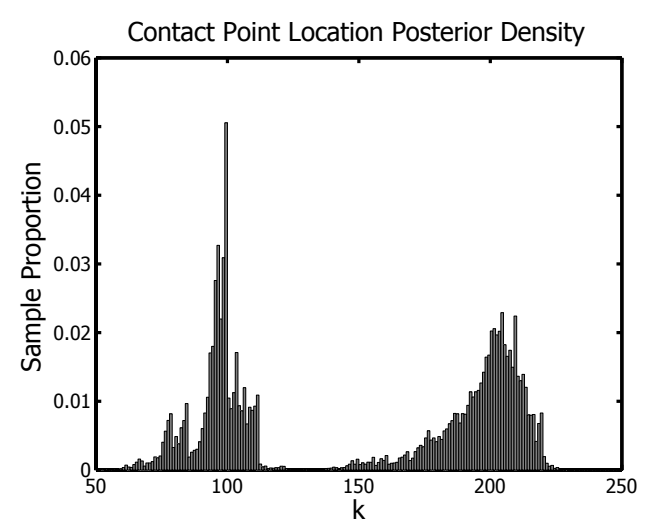

Fig. 5. Experiment 5: Posterior density of the contact point $k$ when the unrestricted prior $k \sim U(1,450)$ is adopted, showing resultant multiple modes

\section{REFERENCES}

[1] C. Rotsch, K. Jacobson, and M. Radmacher, "Dimensional and mechanical dynamics of active and stable edges in motile fibroblasts investigated by using atomic force microscopy," Proc. Natl. Acad. Sci. USA, vol. 96, pp. 921-926, 1999.

[2] A. E. Kerdok, M. P. Ottensmeyer, and R. D. Howe, "Effects of perfusion on the viscoelastic characteristics of liver," J. Biomech., vol. 39, pp. 2221-2231, 2006.

[3] K. D. Costa, "Single-cell elastography: Probing for disease with the atomic force microscope," Disease Markers, vol. 19, pp. 139-154, 2004.

[4] E. K. Dimitriadis, F. Horkay, J. Maresca, B. Kachar, and R. S. Chadwick, "Determination of elastic moduli of thin layers of soft material using the atomic force microscope," Biophys. J., vol. 82, pp. 2798-2810, 2002.

[5] I. N. Sneddon, "The relation between load and penetration in the axisymmetric Boussinesq problem for a punch of arbitrary profile," Internat. J. Eng. Sci., vol. 3, pp. 4757, 1965.

[6] K. D. Costa, A. J. Sim, and F. C-P. Yin, "Non-Hertzian approach to analyzing mechanical properties of endothelial cells probed by atomic force microscopy," ASME J. Biomech. Eng., vol. 128, pp. 176-184, 2006.

[7] B. P. Carlin, A. E. Gelfand, and A. F. M. Smith, "Hierarchical Bayesian analysis of changepoint problems," Appl. Stat., vol. 41, no. 2, pp. 389-405, 1992.

[8] E. J. Chen, J. Novakofski, W. K. Jenkins, and Jr. W. D Brien, "Young's modulus measurements of soft tissues with application to elasticity imaging," IEEE Trans. Ultrason. Ferroelectr. Freq. Control, vol. 43, pp. 191194, 1996. 